\title{
Too much of a good thing? Overdiagnosis, or overestimating risk in preventive genomic screening
}

\author{
Karen M Meagher ${ }^{1}$ \& Jonathan S Berg*,2 \\ ${ }^{1}$ Department of Social Medicine, University of North Carolina at Chapel Hill, Chapel Hill, NC 27599-7240, USA \\ ${ }^{2}$ Department of Genetics, The University of North Carolina at Chapel Hill, Chapel Hill, NC, 27599-7264, USA \\ *Author for correspondence: +1 919966 7043; jonathan_berg@med.unc.edu \\ "Harris and colleagues propose replacing categorical thinking such as 'disease' and 'predisease'
with a broader concept they dub 'predictor of poor health' (PPH) that is meant to capture
probabilistic understanding of an adverse health event that offers a candidate for prevention"
}

First draft submitted: 7 May 2018; Accepted for publication: 17 May 2018; Published online:

27 September 2018

Keywords: medical genetics $\bullet$ medicalization $\bullet$ overdiagnosis $\bullet$ precision health $\bullet$ predictor of poor health $\bullet$ prevention $\bullet$ preventive genomic screening

\section{Forward}

Preventive screening for rare, actionable monogenic disorders is among the armamentarium of tools that might be useful for personalized patient care. Currently, genetic testing is clinically indicated for patients with a strong personal or family history of heritable disease, or related symptoms [1]. However, these tests (even genome-scale sequencing) are increasingly being offered to asymptomatic patients without clinical indications - and consequently the list of potential results from these tests is expanding dramatically. As such efforts scale up, they can begin to approximate proposals for genomic screening in the general population [2]. Although typically articulated in terms of personalized benefits to individual patients, the underlying approach to early detection also permits precision initiatives to target interventions to high-risk groups. Thus, the programmatic success of preventive genomic screening (PGS) can be evaluated in terms of both personalized and population health outcomes. However, overdiagnosis, a complex phenomenon that represents a downside of any population screening endeavor, requires increased scrutiny in this new context.

\section{A cautionary tale}

Conceptually, there is no settled definition of overdiagnosis, a matter complicated by related concepts such as misdiagnosis, false positives and overtreatment [3,4]. Overdiagnosis has been defined as diagnosis that would otherwise not go on to cause symptoms or death' (p. 605) [5] or as giving a diagnosis that does not yield a net benefit [6]. Moreover, the notion of overdiagnosis is conceptually mismatched to preventive genomics, which is not always considered diagnostic per se, but rather detects genetic variants that confer greater risk of morbidity or mortality [7]. We therefore sought to explore the concept of overdiagnosis in the setting of preventive genomic screening.

The history of other screening efforts suggests that concerns about overdiagnosis should be heeded before adopting widespread PGS programs. For example, some estimates indicate that $23-42 \%$ of prostate cancer cases detected through prostate-specific antigen screening in the USA are overdiagnoses [8], because they would never have been detected clinically or symptomatically in the absence of screening. In some cases, cancers grow so slowly that the patient would never have experienced symptoms, while in other cases the patient is already ill or becomes sick from a different condition and dies as a result [8]. In these cases, screening simply does not clinically benefit such patients. Worse yet, screening can be detrimental when it leads to downstream interventions, such as biopsies or aggressive treatments. As a result, some patients may shoulder significant burdens of both screening and subsequent interventions, without experiencing health benefits to offset these harms. 


\section{Genomic screening, predisease \& drivers of overdiagnosis in genetic disorders}

Hofmann notes that concerns about overdiagnosis often reflect a bundle of related problems for screening programs that need unpacking [4]. For example, consider how such worries have been posed as an important ethical consideration in testing for autosomal dominant polycystic kidney disease (ADPKD) among children at risk due to family history [9]. Previously considered an adult-onset condition, evidence suggests that symptoms can be detected at an earlier age among those with deleterious ADPKD variants. However, detecting at-risk children might come at the cost of labeling and potentially subjecting healthy children to increased scrutiny, given the wide variability in the progression of the condition [9]. Importantly, the ADPKD case demonstrates that overdiagnosis is a concern in preventive clinical care within at-risk families, a worry that could be heightened by moving to the general population.

What are the sources of overdiagnosis in PGS? Pathirana and colleagues performed a scoping review to identify general drivers of overdiagnosis and suggested five domains: culture; the health system; industry; professionals; and patients and the public [10]. A full discussion of each of these domains is beyond the scope of this commentary. Nevertheless, implementing PGS in a responsible fashion should involve careful consideration of the evidence, promoting societal benefit and minimizing individual harms [11]. Shared decision-making in light of patient attitudes is also relevant, as screening decisions can be highly preference-sensitive, reflecting patients' underlying values [12].

We will set aside for now the concern about 'false positive' results, which can be the result of technical errors (sample swaps or sequencing artifacts) or interpretive errors (such as incorrectly asserting pathogenicity when in fact a variant is not causal for a monogenic condition). These false positives clearly constitute a serious constraint on positive predictive value in genomic screening, where the prior probability that an individual has a given genetic disorder is very low and our ability to properly interpret variants remains substantially suboptimal [13,14]. Even assuming that these concerns were minimal, we are still faced with two fundamental and intertwined facets of every monogenic condition: penetrance and expressivity. Penetrance is a measure of the proportion of patients with a pathogenic variant who will develop symptoms of the condition, while expressivity represents the range of manifestations seen among individuals with a given condition (age of onset, severity of disease, presence or absence of specific subphenotypes, etc.). We propose that incomplete penetrance and variable expressivity, both of which are likely underestimated in the current literature, comprise two specific sources of overdiagnosis in PGS. These facets of underlying genetic disease etiology reflect complex interactions with social determinants of health, age, sex and comorbid medical conditions that govern how many of those who screen positive will ultimately benefit from early detection.

A scaled up PGS program would situate these facets in a societal context that leads to medicalization of predisease stages. 'Predisease' labels often arise as a result of screening, capturing instances in which there is no sharp line demarcating the healthy from the sick [15] - such as precancerous lesions or borderline elevations in blood pressure or glucose levels $[15,16]$. Similarly, PGS positive findings will prompt a cascade of downstream evaluations, including detailed family history gathering, further diagnostic tests, surveillance, behavior modification recommendations or even invasive preventive interventions. A clinical diagnosis may be established in some who receive follow-up evaluations in the wake of genomic screening, but for many such findings there may not be a gold-standard confirmatory test or a means of determining who is more likely to develop symptoms, or when. The rise of prediseases reflects the convergence of experiences of patients who are at-risk with the experiences of patients with the condition itself [17]. The prospect of viewing deleterious genetic variants as prediseases is not farfetched: the hereditary breast and ovarian cancer genetic advocacy group FORCE has self-described their own members as 'previvors' [18].

\section{Furthering evidence-based PGS}

As described here, overdiagnosis underscores the potential for PGS to fall flat due to the identification of genetic predispositions that do not reliably track current or future harms to health. Harris and colleagues propose replacing categorical thinking such as 'disease' and 'predisease' with a broader concept they dub 'predictor of poor health' $(\mathrm{PPH})$ that is meant to capture probabilistic understanding of an adverse health event that offers a candidate for prevention $[19,20]$. Using this framework, overdiagnosis can be viewed as inappropriately approaching treatment or monitoring for a person with a weak PPH the same way we would treat a person with a strong PPH [19,20]. Screening should aim for detection of those with strong PPH [20] while the recommended strategy for those with weak PPHs should exclude invasive interventions and potentially even eschew increased surveillance. In PGS, calculating PPH 
would include not only the degree of certainty on the part of the variant interpretation but also the penetrance and expressivity of the condition, each of which serve as variables in predicting manifestations for any given condition.

PGS offers the tantalizing prospect of forestalling disease progression by stratifying patients into precise risk groups. Yet the combined confusion resulting from incomplete penetrance and variable expressivity in a context prone to medicalization demands serious consideration of the question: how many people will have a genetic 'predisease' that never leads to symptoms, for every person that is considered to be saved from disease or death? [4]. The population health consequences of detecting genetic risks are likely to be understood in hindsight, when health outcomes for cohorts who do and do not receive PGS are retrospectively compared. As a result, at the advent of genomic screening, clinicians will encounter situations of uncertainty in which an intervention's individual risk-benefit profile for an asymptomatic patient is unknown. Moreover, the future is likely to hold more of the same challenges that prostate-specific antigen screening currently encounters, where determining the wisdom of screening for an individual patient involves a nuanced reconciling of population screening guidelines with clinical judgment and patient attitudes.

Acknowledgements

We would like to thank JP Evans, R Harris and GE Henderson for their comments on an earlier draft of this paper.

Authors' contributions

This manuscript was prepared in accordance Good Publication Practice (GPP) guidelines and all contributors met International Committee of Medical Journal Editors (ICMJE) criteria for authorship. Authors are listed in order of lead and senior author, based on their contributions. KM Meagher and JS Berg jointly developed an initial outline. KM Meagher reviewed the conceptual and bioethical literature on overdiagnosis and wrote the first draft. JS Berg reviewed the overdiagnosis and medical genetics literature and substantially revised and expanded the second draft. All authors critically reviewed and provided substantial revisions to subsequent iterative drafts, approved the final version for submission, and agreed to be accountable for all aspects of the work.

Financial \& competing interests disclosure

Support for the preparation of this article was provided by the US National Human Genome Research Institute (NHGRI) under (grant number NIH P50 HG004488) (G Henderson, principal investigator) 'Center for Genomics and Society' (CGS) 6/2013-5/2018. The authors have no relevant affiliations or financial involvement with any organization or entity with a financial interest in or financial conflict with the subject matter or materials discussed in the manuscript. This includes employment, consultancies, honoraria, stock ownership or options, expert testimony, grants or patents received or pending, or royalties.

No writing assistance was utilized in the production of this manuscript.

\section{References}

Papers of special note have been highlighted as: • of interest; $\bullet \bullet$ of considerable interest

1 Prevention CFDCA. Inside knowledge: get the facts about gynecologic cancer (2018). https://www.cdc.gov/cancer/knowledge/provider-education/genetics/genetic-counseling-testing.htm

2 Adams MC, Evans JP, Henderson GE, Berg JS. The promise and peril of genomic screening in the general population. Genet. Med. 18(6), 593-599 (2016).

3 Jenniskens K, De Groot JAH, Reitsma JB, Moons KGM, Hooft L, Naaktgeboren CA. Overdiagnosis across medical disciplines: a scoping review. BMJ Open 7(12), e018448 (2017).

4 Hofmann B. Diagnosing overdiagnosis: conceptual challenges and suggested solutions. Eur. J. Epidemiol. 29(9), 599-604 (2014).

-• Conceptual and semantic analysis of overdiagnosis and proposal of differentiated nomenclature.

5 Welch HG, Black WC. Overdiagnosis in cancer. J. Natl Cancer Inst. 102(9), 605-613 (2010).

6 Carter SM, Rogers W, Heath I, Degeling C, Doust J, Barratt A. The challenge of overdiagnosis begins with its definition. BMJ 350, h869 (2015).

7 Murray MF. Your DNA is not your diagnosis: getting diagnoses right following secondary genomic findings. Genet. Med. 18(8), 765-767 (2016).

8 Loeb S, Bjurlin M, Nicholson J et al. Overdiagnosis and overtreatment of prostate cancer. Eur. Urol. 65(6), 1046-1055 (2014).

9 Harris T. Is it ethical to test apparently "healthy" children for autosomal dominant polycystic kidney disease and risk medicalizing thousands? Front. Pediatr. 5, 291 (2017).

10 Pathirana T, Clark J, Moynihan R. Mapping the drivers of overdiagnosis to potential solutions. BMJ 358, j3879 (2017)

11 Cho MK. Preventive genomic sequencing in the general population: do PGS fly? Am. J. Bioeth. 15(7), 1-2 (2015). 
12 Ubel PA, Scherr KA, Fagerlin A. Empowerment failure: how shortcomings in physician communication unwittingly undermine patient autonomy. Am. J. Bioeth. 17(11), 31-39 (2017).

13 Vassy JL, Christensen KD, Schonman EF et al. The impact of whole-genome sequencing on the primary care and outcomes of healthy adult patients: a pilot randomized trial. Ann. Intern. Med. 167(3), 159-169 (2017).

14 Aronson SJ, Clark EH, Varugheese M, Baxter S, Babb LJ, Rehm HL. Communicating new knowledge on previously reported genetic variants. Genet. Med. 14(8), 713-719 (2012).

15 Viera AJ. Predisease: when does it make sense? Epidemiol. Rev. 33, 122-134 (2011).

16 Jutel A, Nettleton S. Toward a sociology of diagnosis: reflections and opportunities. Soc. Sci. Med. 73(6), 793-800 (2011).

- Overview of a special issue dedicated to sociological analysis of diagnosis, encompassing different sociological methods. This article distinguishes diagnosis-as-category, diagnosis-as-process and consequences of diagnosis.

17 Aronowitz RA. The converged experience of risk and disease. Milbank Q. 87(2), 417-442 (2009).

18 Friedman S. Previvor: past, present, \& future. (2016) http: //www.facingourrisk.org/get-involved/HBOC-community/BRCA-HBOC-blogs/FORCE/hboc-week/previvor-past-present-future/

19 Harris R. Overview of screening: where we are and where we may be headed. Epidemiol. Rev. 33, 1-6 (2011).

20 Harris R, Sawaya GF, Moyer VA, Calonge N. Reconsidering the criteria for evaluating proposed screening programs: reflections from 4 current and former members of the U.S. Preventive Services Task Force. Epidemiol. Rev. 33, 20-35 (2011).

-. The authors revisit the 1968 Wilson and Jungner principles for evaluating all screening programs and suggest reframing the target of screening in terms of 'predictors of poor health.' 